\title{
Immunotherapy Against Acetylated Tau at K280 Ameliorates Behavioral Impairments and Pathologies of Tau Transgenic Mouse
}

Na-Young Kim

University of Ulsan College of Medicine

Ha-Lim Song

University of Ulsan College of Medicine

Kwangmin Cho

University of Ulsan College of Medicine

Young-Lim Shim

University of Ulsan College of Medicine

Kyounghye Lee

University of Ulsan College of Medicine

Yeon-Seon Mun

University of Ulsan College of Medicine

Seung-Eun Lee

University of Ulsan College of Medicine

Min-Seok Kim

University of Ulsan College of Medicine

Hyun-Ju Kim

University of Ulsan College of Medicine

Dong-Hou Kim

University of Ulsan College of Medicine

Seung-Yong Yoon ( $\nabla$ ysy@amc.seoul.kr)

University of Ulsan College of Medicine

\section{Research article}

Keywords: immunotherapy, Alzheimer's disease, $\beta$-amyloid, tau, acetylation

Posted Date: November 4th, 2020

DOI: https://doi.org/10.21203/rs.3.rs-100198/v1 
License: (c) (i) This work is licensed under a Creative Commons Attribution 4.0 International License. Read Full License 


\section{Abstract}

Background: Tau is gaining attention as a target of therapeutic antibodies for Alzheimer's disease (AD) and other tauopathies but it was recently of debate which epitope on tau has therapeutic potency. While the hexapeptide ${ }^{275}$ VQIINK ${ }^{280}$ is a critical region for tau aggregation and K280 is acetylated in various tauopathies including AD, there has been no immunotherapy targeting acetylated K280.

Methods : Acetylated K280 was first chosen as therapeutic target from small pilot studies comparing in vivo behavioral efficacy of tau transgenic mice by immunizing with several putatively pathogenic epitopes such as acetylated or phosphorylated or cleaved tau.

Results : Therapeutic efficacy in tau transgenic mice was further evaluated with behavior, pathology and biochemical experiments after either active immunization with tau peptide acetylated at K280 or passive immunization with anti-tau antibody targeting K280 acetylation. Vaccination with tau peptide acetylated at K280 or antibody (Y01) targeting it ameliorated memory impairments and pathology in tau transgenic mice via intracerebroventricular or intraperitoneal route.

Conclusions: This study showed that acetylated K280 tau is a good therapeutic target and Y01 could be a novel therapeutic antibody for $A D$ and other tauopathies.

\section{Background}

Tau protein aggregates, such as neurofibrillary tangles (NFTs), are a key pathological finding in Alzheimer's disease (AD) and other neurodegenerative disorders, collectively referred to as tauopathies. In $A D$, tau pathology has a hierarchical pattern of accumulation that first appears in the transentorhinal cortex and subsequently spreads to the hippocampus and neocortex(Braak and Braak, 1991). The spread of tau pathology is underscored by cell-to-cell transmission of pathological tau species(de Calignon et al., 2012; Michel et al., 2014; Wu et al., 2016; Clavaguera et al., 2009), which may act as targets of therapeutic antibodies. However, it is unclear which species and distinct features of tau are critical for pathological tau propagation. Tau is expressed as six isoforms with zero, one, or two N-terminal acidic inserts $(0 \mathrm{~N}, 1 \mathrm{~N}$, or $2 \mathrm{~N}$, respectively) and either three or four microtubule-binding repeats (MTBR) (3R or 4R) (Goedert et al., 1989). Tau (2N4R) is a long protein consisting of 441 amino acids and can be modified by posttranslational modifications such as phosphorylation, acetylation, and cleavage at various epitopes. This makes it more challenging to select the most effective epitope for tau in contrast to short $\beta$-amyloid (A $\beta$ ). Hence, the roles and relationships of these various modified epitopes in AD pathogenesis should be carefully considered.

Tau is acetylated at lysine residues in the MTBR, hence providing a novel regulatory modification other than phosphorylation (Irwin et al., 2012; Min et al., 2010; Cohen et al., 2011). Tau acetylation impairs normal interaction with microtubules, prevents physiological tau-mediated stabilization of microtubules, and induces formation of pathological tau aggregates (Irwin et al., 2012; Min et al., 2010; Cohen et al., 2011). Acetylation of tau at lysine-280 (K280) is increased in AD, corticobasal degeneration (CBD), 
progressive supranuclear palsy (PSP), and several frontotemporal dementia with Parkinsonism (FTDP)-17 familial cases of dementia(Cohen et al., 2011) but is rarely observed in control brain tissues(Irwin et al., 2012), which delineates the disease relevant nature of K280 acetylation. Deletion of lysine-280 ( $\triangle \mathrm{K} 280)$ of tau is reported in frontotemporal dementia with Parkinsonism (FTDP)-17 and AD (Momeni et al., 2009; Rizzu et al., 1999), and $\Delta K 280$ facilitates $\beta$-structure formation and accelerates tau aggregation in in vitro and in vivo models (Mocanu et al., 2008), hence the hexapeptide ${ }^{275}$ VQIINK $^{280}$, encompassing K280, is well-known as a critical region for tau aggregation (Mukrasch et al., 2005). However, there has been no immunotherapy developed targeting acetylated K280 (acK280).

Various tau species and modifications have been reported to be related to $A D$, but it is unclear which forms are true therapeutic targets rather than merely being pathologically correlated findings. Here, we addressed this with small pilot studies comparing in vivo behavior efficacy of tau transgenic mice by immunizing with several putatively pathogenic epitopes such as acetylated or phosphorylated or cleaved tau, then chose acK280 as a target. We validated therapeutic efficacy targeting tau-acK280 with active immunization or antibody in tau transgenic mice.

\section{Materials And Methods}

\section{Animal models}

Studies were performed in C57BL/6J-wild type, Tau-P301L-transgenic, and Tau-P301S-transgenic mice. Tau-P301L mice were generated from JNPL3 (Tau-0N4R, P301L) mice purchased from Taconic, Inc. (NY, USA) and backcrossed to C57BL/6J over five generations. Tau-P301S mice were generated from PS19 (Tau-1N4R, P301S) mice purchased from Jackson Laboratories, Inc. (Bar Harbor, ME, USA) and backcrossed to $\mathrm{C} 57 \mathrm{BL} / 6 \mathrm{~J}$ over five generations. All mouse genotyping was confirmed by PCR. The mice were caged ( 3 to 5 mice per cage) and kept on a 12h light/dark cycle at a constant ambient temperature $\left(22 \pm 1^{\circ} \mathrm{C}\right)$ with $40-60 \%$ humidity. Food and water were available ad libitum. All animal protocols were approved by Asan Institute for Life Science Animal Experimentation Committee.

\section{Peptides}

The peptide immunogen Tau 226-236 (phosphorylated tau threonine 231), Tau 275-286 (acetylated tau lysine 280), Tau 303-316 (acetylated tau lysine 311), and Tau 381-391 (cleaved tau glutamic acid 391) were synthesized at Anygen (Nam-myun, KOREA). N-terminal was conjugated with Keyhole Limpet Hemocyanin (KLH). The peptide was purified by Shimadzu HPLC 10AVP system (Purity $91.8 \%$ ) with a 5$65 \%$ linear gradient of acetonitrile in $0.05 \%$ Trifluoroacetic acid (TFA).

\section{Generation of hybridoma cells}


Mouse hybridoma cells were manufactured by Young-In Frontier Co., Ltd. (Seoul, Korea). Briefly, the antigen was mixed with Complete Freund adjuvant (Sigma-Aldrich, St. Louis, MO, USA) and intraperitoneally injected into female BALB/c mice. After 2 weeks, serum was collected from mice and antibody titer was measured using ELISA. The antigen was mixed with PBS and injected into mice with a second injection for boosting. After 3 days, the spleen was extracted. After washing the tissue with culture medium, cells were separated. Myeloma cells (Sp2/0Ag14) were fused with cells separated from mice using polyethylene glycol (PEG) (Roche Life Science, Mannheim, Germany). Fused cells were cultured using 1XHAT culture medium (Sigma-Aldrich, St. Louis, MO, USA). Using a HT culture medium (Gibco Life Technologies, NY, USA), cells were pipetted into a 96-well plate. Hybridoma cells were cultured for 7-10 days. For positive clone screening, hybridoma supernatants were screened by ELISA for reactivity with $\mathrm{K} 280$-ac peptides. ELISA screens and the cloning process were repeated until the final clone was verified. The best clone was selected and termed ADEL-Y01 antibody.

\section{Preparation of ADEL-Y01 antibody}

Hybridoma cells were grown at $37^{\circ} \mathrm{C}$ in CD Hybridoma Medium (Gibco Life Technologies, NY, USA) supplemented with $8 \%$ glutamax at $1: 25$ dilution, $2 \%$ ultra-low IgG fetal bovine serum (FBS), and $1 \%$ penicillin streptomycin (Gibco). After 1 week, supernatant was collected and centrifuged at 13,000 rpm for $15 \mathrm{~min}$ at $4^{\circ} \mathrm{C}$. Supernatant was filtered with $0.4 \mathrm{~mm}$ filter unit and $1 \mathrm{M}$ Tris-pH 9.0 was added for neutralization. All experiments were performed on AKTA-start chromatography system (GE Healthcare Life Sciences, Piscataway, NJ, USA). The equipment was washed with distilled water, and the column was connected. The column was equilibrated with 1X Phosphate-Buffered Saline (PBS), and the sample was allowed to flow. Once the sample was exhausted, a wash with 1XPBS was performed, and the antibody was eluted using $0.1 \mathrm{M}$ glycine $(\mathrm{pH}$ 2.7). The purified antibody was neutralized with $1 \mathrm{M}$ Tris $\mathrm{pH}$ 9.0. The antibody was dialyzed with 1XPBS. The band was checked using Coomassie blue staining. The Y01 antibody was stored at $-20^{\circ} \mathrm{C}$.

\section{Active immunization}

At 3 months of age, male transgenic P301L mice and C57BL/ 6 mice were immunized with either peptide or Adju-Phos adjuvant (InvivoGen, San Diego, USA) only. Mice were intraperitoneally injected with $50 \mathrm{mg}$ peptide mixed 1:1 ( $\mathrm{v} / \mathrm{v}$ ) with Adju-Phos adjuvant (25 mg per mouse). After the first immunization, treatment was administered at intervals of 2 weeks. Injections were performed once a month. After the last injection, mice were subjected to behavioral tests and diffusion tensor imaging (DTI). All mice were sacrificed at 8 months.

\section{Passive immunization}




\section{Intracerebroventricular infusion}

At 8 months of age, C57BL/ 6 and P301L mice were administered intracerebroventricular (icv) injections of immunoglobulin $\mathrm{G}(\mathrm{lgG})$ control or $\mathrm{Y} 01$ antibody $(1.9 \mathrm{mg} / \mathrm{mL})$. The pump implantation surgery was performed according to the manufacturer's instructions. Briefly, before the surgery, an L-shaped infusion cannula was attached to catheter tubing (Alzet, California, USA). A brain infusion kit was attached to a micro-osmotic pump (Alzet, California, USA). The assembly pump was implanted using a stereotactic apparatus (Harvard Apparatus, Massachusetts, USA) into the right lateral ventricle at $0.58 \mathrm{~mm}$ posterior to bregma, $1 \mathrm{~mm}$ lateral to the midline, and $2 \mathrm{~mm}$ from the skull surface. An osmotic pump was subcutaneously implanted into the back of each mouse. Each pump was filled with IgG control or Y01 antibody $(1.9 \mathrm{mg} / \mathrm{mL})$. The osmotic pump delivered antibodies continuously at $0.11 \mu \mathrm{L} / \mathrm{h}$ for 28 days. Reservoir volume was $100 \mu \mathrm{L}$. Behavioral analysis was performed during the last 3 weeks of infusion. At 9 months of age, all mice were sacrificed.

\section{Intraperitoneal injection}

At 7 months of age, C57BL/ 6 and P301L mice were injected intraperitoneally (IP) with IgG control or Y01 antibody $(50 \mathrm{mg} / \mathrm{kg})$. Mice were subjected to behavioral tests. At 10 months of age, all mice were sacrificed. At 14 months of age, C57BL/ 6 and P301L mice were injected IP with IgG control or Y01 antibody $(50 \mathrm{mg} / \mathrm{kg})$. The mice were subjected to behavioral testing. At 17 months of age, all mice were sacrificed. At 5 months of age, C57BL/ 6 and P301S mice were injected IP with two different doses of Y01 antibody ( 5 and $50 \mathrm{mg} / \mathrm{kg}$ ) or $\mathrm{lgG}$ control $(50 \mathrm{mg} / \mathrm{kg}$ ). At 8 months of age, all mice were sacrificed. All mice received injections weekly for 3 months. Behavioral analysis was performed during the last 4 weeks of injection.

\section{Behavioral tests}

\section{Nest-building}

Mice were isolated in home cages a day before testing. Cotton was placed in each cage for the scoring of nesting behavior. The next day, nesting behavior was scored according to the following guidelines: score 1 , cotton rarely touching and $>90 \%$ intact cotton; score 2 , cotton partially ripped with $50-90 \%$ intact; score 3 , cotton ripped with $<50 \%$ intact and spread around the cage; score 4 , cotton mostly ripped with $<10 \%$ intact and gathered into side of the cage; score 5 , cotton almost ripped and perfect nest shape.

\section{Grip strength test}

To measure strength, a weights test was performed. Animals were held by the base of the tail and lowered to the apparatus comprising a coiled wire ball for gripping. Apparatus weight was less than $3 \mathrm{~g}$. 
Time taken to drop the apparatus was measured. All tests were repeated three times.

\section{Vertical grid test}

All groups were subjected to a vertical test. Mice were placed on a wire net (horizontal, $18 \mathrm{~cm}$; vertical, 30 $\mathrm{cm})$. The wire net was turned over, and the time taken for mice to fall was measured. All tests were repeated three times.

\section{Y-maze}

Mice were subjected to a Y-maze test to assess short-term spatial memory. The apparatus was a Yshaped plastic maze with three arms. Mice were habituated for $5 \mathrm{~min}$. One arm ( $Y$-shaped left arm) was open and the other arm ( $\mathrm{Y}$-shaped right arm) was blocked during a 5-min test. After $1 \mathrm{~h}$, the other arm ( $\mathrm{Y}$ shaped right arm) was unblocked and behavior was analyzed for $5 \mathrm{~min}$. Time in novel arm is the time to stay on the opened Y-shaped right arm.

\section{Rotarod}

Mice were placed on an accelerating rotarod, and exercise capacity was measured. The speed was slowly increased from 4 to $40 \mathrm{rpm}$. The time (sec) and speed (rpm) at which mice fell were measured. Mice were trained for 4 days before the test. On the fifth day, motor test was performed. All tests were repeated 3 times.

\section{Water maze}

Mice were subjected to a water maze(Vorhees, 2006). The water pool was divided into four quadrants, NE, North East; SE, South East; SW, South West; NW, North West quadrants. Briefly, all mice were trained to locate a visible platform for 4 days. Training was conducted three times a day and start positions were selected as NE, SE and SW quadrants. On the 5day, the platform was removed, and movement was analyzed, such as time taken to reach the target.

\section{Contextual fear conditioning}

Mice were placed in a context in which they received a foot shock (Hirotaka Shoji1, 2014). On day 1, all mice were habituated to the chamber for $2 \mathrm{~min}$. The sound was presented for 30 seconds paired with a shock up to the last $2 \mathrm{~s}$. This process was repeated three times. On day 2, after sufficient rest, mice were habituated to the context without any stimulation for $3 \mathrm{~min}$. Then, the sound was presented without shock for 3 min, and behavior was analyzed. 


\section{Diffusion tensor imaging}

Diffusion tensor imaging was performed in mouse after acquiring T2 weighted (T2w). All mice were anesthetized with 1-1.5\% isoflurane in a mixture of oxygen and room air gases (1:2) delivered via a nose cone. Respiratory rate, electrocardiogram, and rectal temperature were monitored. Parameters and data processing were performed as previously described ( $\mathrm{H}-\mathrm{J} \mathrm{Kim}, 2017)$. All mouse using a 4 shot spin-echo based echo planar imaging (EPI) sequence with TR/TE $=3750 / 46.22 \mathrm{~ms}, 96 \times 96$ matrix and an encoding scheme of 30 gradient directions at b-value $=1000 \mathrm{~s} / \mathrm{mm}^{2}(\mathrm{H}-\mathrm{J} \mathrm{Kim}, 2017)$. The hippocampus was selected as the ROI. Data were analyzed by fractional anisotropy (FA) and mean diffusivity (MD) values.

\section{Blood sampling}

Blood was collected from the facial vein 1 week before the start of immunization (T0), 1 week after the third injection (T1), 1 week after the fourth injection (T2), and at sacrifice (TF). Blood plasma was harvested by centrifugation, and antibody titer was analyzed.

\section{Enzyme-linked immunosorbent assay}

For measuring plasma titers of antibodies, indirect ELISA was performed. Briefly, 96-half well plates (Costar, Corning Life Sciences, Massachusetts, USA) were coated with either $250 \mathrm{ng} /$ well immunizing peptides diluted in capture solution overnight at $4^{\circ} \mathrm{C}$. After blocking for $1 \mathrm{~h}$, plasma samples were diluted 1:500 in blocking solution and incubated for $2 \mathrm{~h}$. After $2 \mathrm{~h}$ of incubation at room temperature, horseradish peroxidase (HRP)-conjugated secondary antibody was added to each well for $2 \mathrm{~h}$. All steps were followed by three washes with wash solution. Then, samples were processed with 3,3',5,5'-tetramethylbenzidine (TMB) and a microtiter plate reader MAX190 (Molecular Devices, California, USA) calibrated to $450 \mathrm{~nm}$.

\section{Western blot}

Mouse tissues were homogenized in Pro-prep, a protein extraction solution (Intron Biotechnology, Seongnam, Korea), according to the manufacturer's instructions. The suspension was centrifuged, and the supernatant was collected. Concentrations were measured using the Bradford Assay. Proteins were mixed with $4 \mathrm{X}$ sample buffer $(60 \mathrm{mM}$ Tris-HCl [pH 6.8], $2 \%$ [w/v] sodium dodecyl sulfate [SDS], 25\% [v/v] glycerol, $14.4 \mathrm{mM}$ [v/v] b-mercaptoethanol, and bromophenol blue). For semi-denaturation conditions, mouse tissue or cell lysate samples were mixed with $2 X$ laemmli sample buffer without mercaptoethanol. Protein was resolved by SDS-polyacrylamide gel electrophoresis and transferred to polyvinylidenedifluoride membranes (BioRad, California, USA). After $1 \mathrm{~h}$ incubation in blocking buffer, blots were incubated with primary antibodies overnight at $4^{\circ} \mathrm{C}$. Membranes were washed in washing buffer and incubated with horseradish peroxidase-conjugated anti-IgG (Vector Laboratories, California, USA). Membranes were visualized using enhanced chemiluminescence reagents (Thermo Fisher 
Scientific, Rockford, IL, USA) and x-ray film. Band intensities were measured and analyzed with ImageJ software (NIH, Bethesda, MD).

\section{RAB-RIPA-formic acid extraction}

A three step extraction protocol was performed as described previously(Kaoru Yamada and Holtzman, 2015). Briefly, cortex was homogenized with RAB buffer (0.1 M MES, 1 mM EDTA, $0.5 \mathrm{mM} \mathrm{MgSO}_{4}, 750$ $\mathrm{mM} \mathrm{NaCl}, 20 \mathrm{mM} \mathrm{NaF}, 1 \mathrm{mM} \mathrm{Na}$ VO4, protease/phosphatase inhibitors) and centrifuged at 50,000 ' $\mathrm{g}$ for 20 min. The RAB insoluble pellet was solubilized with RIPA buffer $(0.15 \mathrm{M} \mathrm{NaCl}, 50 \mathrm{mM}$ Tris, $0.5 \%$ deoxycholic acid, $1 \%$ Triton X-100, 0.5\% SDS, 25 mM EDTA, pH 8.0, protease/phosphatase inhibitor) and centrifuged at 50,000 ' $\mathrm{g}$ for $20 \mathrm{~min}$. The RIPA insoluble pellet was solubilized with $70 \%$ formic acid.

\section{Immunoprecipitation}

Brain lysates of immunized mice were incubated with $100 \mathrm{~mL}$ of protein G-agarose bead (GE Healthcare Life Sciences, Piscataway, NJ, USA) at $4^{\circ} \mathrm{C}$ overnight. The samples were collected by centrifuging at $6,000 \mathrm{rpm}$ for $1 \mathrm{~min}$ at $4^{\circ} \mathrm{C}$ and washed three times with $0.1 \%$ Triton $\mathrm{X}-100$ in PBS. The samples were boiled in SDS sample buffer and processed by western blot.

\section{Immunohistochemistry}

Frozen mouse brain blocks were sectioned and attached on coated glass slides. Sectioned brain tissues were washed with 1 XPBS and tissues were permeabilized with 1 XPBS containing $0.1 \%$ Triton X-100 for 10 min at RT. The tissues were washed and blocked with PBS containing $3 \%$ bovine serum albumin for 1 $h$ at RT. The tissues were then washed with 1 XPBS and incubated with primary antibody at $4^{\circ} \mathrm{C}$ overnight. After washing with 1XPBS, for DAB staining, a biotinylated secondary antibody (Vector Laboratories, California, USA) was added for $1 \mathrm{~h}$ at RT. Tissues were washed and incubated in an avidin-biotinperoxidase complex (Vector Laboratories, California, USA). For visualization, tissues were incubated for $10 \mathrm{~min}$ in diaminobenzidine (DAB) solution. Tissues were washed and mounted with Canada balsam (Sigma-Aldrich, St. Louis, MO, USA). DAB-reacted images were obtained using a Zeiss microscope (Carl Zeiss, Oberkochen, Germany) and processed using the AxioVision Imaging System.

\section{Antibodies}

In this experiment, following antibodies were used: Y01 antibody (ADEL, Inc.), anti-Tau5 (Invitrogen, AHB0042), anti-AT8 (Thermo, MN1020), anti-pSer396 (Thermo, 44-752G), anti-acetyl-K280 (Anaspec, AS56077), anti-pT231 (Thermo, MN1040), anti-PSD95 (Abcam, ab2723), anti-NMDAR (Chemicon, AB1548), anti-synapsin-1 (Chemicon, MAB355), anti-synaptophysin (Sigma, S5768), anti-Control IgG (Bioxcell, BE0083), and anti- $\beta$-actin (Sigma, A5441). 


\section{Statistical analysis}

All data were analyzed with GraphPad Prism v.5. Data were analyzed using one-way ANOVA (Tukey's post hoc test) or Student's $t$-test. A $p$-value $<0.05$ was considered statistically significant.

\section{Results}

\section{Active immunization with Tau-acK280 is the most effective at reducing behavioral impairments in tau transgenic mice}

Since phosphorylation, acetylation and cleavage of tau are associated with $A D$, we first did a small pilotstudy to test which target we will proceed further for prevention of tau-related impairments, including phosphorylation at T231 (pT231), acetylation at K280 (acK280), acetylation at K311 (acK311), and cleavage at E391 ( $\triangle E 391$ ), as suggested in AD pathogenesis (Additional file 1: Figure S1a). To this end, we immunized tau-P301L transgenic (tau-Tg) mice from 3 to 6 months of age with each keyhole limpet hemocyanine (KLH)-conjugated peptides in aluminum adjuvant and compared their effects on memory and motor impairments (Additional file 1: Figure S1b). Wild-type and control tau-Tg mice received adjuvant alone. When mice were immunized with each type of peptide, they exhibited a robust antibody response against each immunogen, whereas minimal responses were observed in control and tauacK311-immunized mice (Additional file 2: Figure S2). After immunization, we performed behavioral tests including grip strength and vertical tests for motor performance (Additional file 1: Figure S1c, d) as well as Morris water maze tests and fear conditioning (Additional file 1: Figure S1e, f). Only mice immunized with tau-acK280 demonstrated consistent improvement in cognitive and motor performance (Fig. 1, Additional file 3: Figure S3), suggesting that tau-acK280 is the effective therapeutic target for AD and tauopathy relatively than other tau epitopes tested. To further confirm the protective efficacy of tauacK280 immunization, we immunized mice $(n=9)$ with tau-acK280 in an independent experiment (Fig. 1, Additional file 3: Figure S3). Nest building test (Fig. 1A, B), learning and memory tests including the $Y$ maze (Fig. 1C), Morris water maze (Fig. 1D), and motor tests (Additional file 3: Figure S3a, b) revealed impaired cognition and motor performance in tau-Tg mice, but immunization with tau-acK280 attenuated these impairments. In the rotarod test, none of the tau-Tg mice were able to reach $30 \mathrm{rpm}$, but tau-ack280 vaccination fully recovered rotarod performance (64\% of mice) comparable to wild type performance (67\%) (Additional file 3: Figure S3c, d).

\section{Active immunization with Tau-acK280 ameliorates pathological changes in tau-Tg mice}

After immunization for about 4 months, diffusion tensor imaging (DTI) was performed to analyze changes in brain pathology in vivo. DTI analysis showed decreased fractional anisotropy (FA) and mean diffusivity (MD) in the hippocampus of tau-Tg mice (Additional file 4: Figure S4), implying microstructural 
anomalies and decreased white matter integrity. However, these effects were normalized by tau-acK280 immunization (Additional file 4: Figure S4), implying recovery of microstructural anomalies and functional connectivity by immunization. Western blot data revealed an increase in abnormally phosphorylated tau in tau-Tg mice, but this effect was decreased after tau-acK280 immunization (Fig. 2A, B). Acetylated tau was increased in tau-Tg mice, but decreased after tau-acK280 immunization (Fig. 2A, B). The levels of synaptic proteins such as PSD95, NMDA receptors, synaptophysin, and synapsin-1 were decreased in the brains of tau-Tg mice (Fig. 2C, D), indicating impaired synapse structure and function. However, these changes were mostly recovered by tau-acK280 immunization (Fig. 2C, D), implying the protective effects of immunization. Increased accumulation of phosphorylated tau in tau-Tg mice and its normalization by tau-acK280 immunization was also observed using immunohistochemistry (Fig. 2E, F).

\section{Intracerebroventricular infusion of anti-tau-acK280 antibody, Y01, ameliorates cognitive and pathology in tau-Tg mice}

To test whether the anti-tau-acK280 antibody, Y01, had therapeutic potential similar to that of tau-acK280 active immunization (Fig. 1, 2), continuous intracerebroventricular (icv) infusion of Y01 antibody into the lateral ventricle of 8-month-old tau-Tg mice was performed for a month (Fig. 3A). As per the results of active immunization, passive icv immunization with Y01 antibody produced improvements in behavioral tests including nest building test, Y-maze, water maze test, and grip strength test (Fig. 3B-D, Additional file 5: Figure S5a). To confirm characterize the aggregate status of tau in the brain, sequential biochemical extraction was performed with RAB (aqueous buffer), radio immunoprecipitation assay (RIPA) (detergent buffer), and $70 \%$ formic acid (FA) to solubilize the final pellet. Insoluble tau was increased in tau- $\mathrm{Tg}$ mouse brains and was reduced by Y01 antibody infusion (Fig. 3E). Western blots with cortex lysates revealed that total tau (tau-5), tau-pS396, and tau-acK280 levels were decreased in antibody-infused brains (Fig. 3F, G). Immunohistochemistry revealed that increased phosphorylated tau in tau-Tg mice was normalized by Y01 antibody (Fig. 3H, I). The levels of synaptic proteins such as PSD95 and synapsin-1 were decreased in the brains of tau-Tg mice, which showed a trend to be reversed by Y01 antibody, although this did not reach statistical significance (Additional file 5: Figure S5b, c), implying protective effects of immunization.

\section{Systemic administration of Y01 antibody ameliorates behavioral deficits and pathological changes in tau-Tg mice}

To assess whether peripheral administration of the antibody produced similar effects to those of icv infusion, Y01 antibody was injected intraperitoneally (i.p.) (Fig. 4A). Similar to the results of icv infusion (Fig. 3), improvements in behavioral tests including nest building test, Y-maze, and Morris water maze test (Fig. 4B-D) were observed following weekly i.p. injection with Y01 antibody for 3 months starting from 7 or 14 months of age (Fig. 4). To investigate whether the antibody entered the brain and bound to antigens, brain samples were perfused systemically with cold PBS at sacrifice to remove any blood from 
the brain and were then incubated with protein-G-sepharose (PGS) to pull down the antibodies in brain parenchyma. Western blots with anti-mouse IgG antibody revealed that both control IgG and Y01 antibody were present in the brain (Fig. 4E), confirming that antibodies entered the brain even though the blood-to-brain ratio may have been low. Tau protein acetylated at K280 was also pulled down alongside PGS only in Y01 antibody-injected mouse brains (Fig. 4E), indicating that peripherally injected Y01 antibody entered the brain and bound to the antigen, tau-acK280. Semi-denatured western blots with cortex lysates either from mice of 10 or 17 months of age revealed that total tau (tau-5), tau-pT231, and tau-acK280 aggregate levels were decreased in antibody-injected brains (Fig. 4F, G). The levels of synaptic proteins such as PSD95 and synapsin-1 were decreased in the brains of tau-Tg mice, which showed a trend to be reversed by Y01 antibody, although this did not reach statistical significance (Additional file 6: Figure S6a, b), implying protective effects by immunization. To further evaluate the efficacy of Y01 in a different tau-Tg strain, Y01 antibody was injected i.p. to tau-P301S mice at two different doses ( $5 \mathrm{mg} / \mathrm{kg}, 50 \mathrm{mg} / \mathrm{kg}$ ) for 3 months starting from 5 months of age (Fig. $5 \mathrm{~A}$ ). Improvements in the water maze test were observed in the group that was administered $50 \mathrm{mg} / \mathrm{kg}$ of $\mathrm{Y} 01$ (Fig. 5B). Semi-denatured western blots with hippocampus lysates which were fractionated into formic acid from 8 months of age revealed that levels of total tau (tau-5) and tau-acK280 monomers and oligomers/aggregates were decreased in the brains of mice injected with $50 \mathrm{mg} / \mathrm{kg}$ of Y01 (Fig. 5C, D).

\section{Discussion}

There has been several anti-tau antibodies developed for AD and most of them target phosphorylated or non-modified tau(Sigurdsson, 2018). We here for the first time provide anti-acetylated tau antibody as a potential $A D$ therapeutic pipeline, which tau acetylation has been widely suggested to be a critical pathogenic modification in $A D$ (Kontaxi et al., 2017).

p300 acetyltransferase acetylates tau (Min et al., 2010), and p300 acetyltransferase activity is increased in AD brains according to severity (Wong et al., 2013; Aubry et al., 2015) and in tau-Tg mice brains (Min et al., 2015). Phosphorylated $p 300$, the active form of $p 300$, was observed in neurites and cytoplasm of neurons in AD hippocampus and frontal cortex (Wong et al., 2013; Aubry et al., 2015). Pathological activation of p300 inhibits autophagic flux leading to increased tau secretion (Chen et al., 2018). In AD brains, this may aggravate the pathological secretion and propagation of K280-acetylated tau. The pilot results of in vivo epitope screening (Additional file 1: Figure S1) underscore the importance of selecting the right epitopes to achieve therapeutic efficacy. Among our tested epitopes, pT231, $\triangle E 391$, acK280, and acK311, acK280 was the most therapeutically efficient epitope (Additional file 1: Figure S1). Since the efficiency of antibody formation against acK311 was too low (Additional file 2: Figure S2), we were unable to conclude that this epitope was a poor therapeutic target. However, this result may reflect the practical difficulties in developing vaccines or antibodies targeting this epitope. Although we tried to select the therapeutically best epitope, our in vivo screening experiments have limitations that it is a small number pilot study and only covered a subset of AD-related pathological modifications of tau. Hence, other more therapeutically effective tau epitopes may exist. Nevertheless, acK280 is an attractive target because it is an AD-related modification and promotes tau aggregation (Cohen et al., 2011); further, the 
hexapeptide ${ }^{275}$ VQIINK ${ }^{280}$ encompassing K280 residue is critical for tau aggregation (Mukrasch et al., 2005).

Tau protein amount in total cell lysates or tau aggregates were decreased by acK280 immunization or Y01 administration (Fig. 2-5), accompanied by decrease of acetylated or phosphorylated tau. This reduction of total tau or tau aggregates may contribute to the neuroprotective effect of targeting TauacK280. We think that potential mechanisms of tau reduction by Y01 include 1) inhibiting tau aggregation and propagation and 2) enhancing microglial tau clearance since we observed these effects in cell cultures (data not shown). Total tau amount in tau-Tg mice is a steady-state level of tau production, degradation and accumulation. Increased production of pathogenically mutated tau, P301L or P301S, in tau-Tg may hamper normal degradation of tau, augmenting a pathogenic release of aggregated tau and propagation to next neurons. This subsequently aggravates tau aggregation in next neurons increasing the half-life of tau, which may be a vicious cycle of tau accumulation. Anti-tau antibody like Y01 may stop this vicious cycle leading to decrease of total tau amount or tau aggregates by inhibiting propagation or aggregation or by enhancing tau clearance by microglia.

The structure of tau fibrils extracted from the $A D$ brain at atomic resolution has recently been described(Fitzpatrick et al., 2017). Paired helical filaments (PHFs) and straight filaments (SFs) share a common C-shaped filament core comprising a series of $\beta$-sheets from R3 and R4 domains. K280 residue and the rest of R2 reside in the fuzzy coat and not in the fibril's core. Hence, the K280 residue does not seem to be hindered from access by the ADEL-Y01 antibody. The finding that the R2 domain is not in the fibril's core is surprising because the ${ }^{275}$ VQIINK ${ }^{280}$ segment is well-known to be important for tau aggregation (Mukrasch et al., 2005). However, filament structures in the AD brain only represent the end product of tau aggregation, which does not exclude the involvement of other parts of the protein in the initial formation and progression of filaments. Eisenberg's team recently reported the structure of the ${ }^{275}$ VQIINK ${ }^{280}$ segment forming a tight steric zipper with strong intermeshed teeth to confer ${ }^{275}{ }^{V Q I I N K}{ }^{280}$ with greater potency for tau aggregation than that of ${ }^{306}$ VQIVYK $^{311}$ (Seidler et al., 2018). Inhibitors based on the structure of the ${ }^{306}$ VQIVYK ${ }^{311}$ segment only partially inhibit full-length tau aggregation and are ineffective at inhibiting seeding by full-length fibrils. However, structurally designed ${ }^{275}$ VQIINK ${ }^{280}$ inhibitor more potently inhibits tau aggregation and seeding induced by tau fibrils (Seidler et al., 2018). Hence, they suggest that the fibril core itself is not the primary driver of seeding; rather, it serves as a scaffold that clusters ${ }^{275}$ VQIINK ${ }^{280}$ segments together in the fuzzy coat, thereby poising the ${ }^{275}$ VQIINK ${ }^{280}$ steric zippers for seeding. They also suggest that ${ }^{275}$ VQIINK ${ }^{280}$ localization to the fuzzy coat would make it more accessible to protein monomers in the cellular milieu, which in turn explains its prominent role in seeding and exquisite sensitivity to inhibition. Thus, ADEL-Y01 antibody could access the K280 residue which is critical for aggregation and seeding in the fuzzy coat and inhibit these pathogenic mechanisms.

\section{Conclusions}


Several therapeutic anti-tau antibodies are tested in clinical trials and some controversial issues have been raised about which epitope should be targeted in AD or other tauopathies (Courade et al., 2018). We anticipate Y01, the first antibody targeting tau-acK280 of the key aggregation site ${ }^{275}$ VQIINK ${ }^{280}$, would be a new therapeutic pipeline in the field.

\section{Abbreviations}

AD: Alzheimer's disease; NFTs: Neurofibrillary tangles; PHFs: Paired helical filaments ; MTBR: Microtubule-binding repeats; SFs: Straight filaments (SFs); Aß: $\beta$-amyloid; K280: Tau at lysine-280; acK280: Acetylated K280; CBD: Corticobasal degeneration; PSP: Progressive supranuclear palsy; FTDP: Frontotemporal dementia with Parkinsonism

\section{Declarations}

\section{Acknowledgements}

Not applicable.

\section{Author's contributions}

N.Y.K. designed the majority of the experiments, performed the in vivo and ex vivo experiments, analyzed data, and wrote the manuscript. H.L.S. managed animals and reviewed the manuscript. K.M.C. performed the fractionation. Y.L.S. performed analyzed data. K.H.L. managed animals. Y.S.M., S.E.L. and M.S.K. reviewed the manuscript. H.J.K. performed the immunohistochemistry. D.H.K. supervised the project and manuscript review. S.Y.Y. supervised the project, formulated the hypothesis, designed the experiments, and wrote the manuscript. All authors read and approved the manuscript.

\section{Funding}

This study was supported by the Basic Science Research Program of the Ministry of Science, ICT and Future Planning [grant nos. 2018R1A2A1A05077403 and 2017R1A2B2010901].

\section{Availability of data and materials}

All data generated or analyzed during this study are included in this article and its supplementary information files.

\section{Ethics approval}


All animal protocols were approved by Asan Institute for Life Science Animal Experimentation Committee.

\section{Consent for publication}

All authors read and approved the final manuscript.

\section{Competing interests}

S.Y.Y. founds ADEL, Inc; S.Y.Y., D.H.K., N.Y.K., K.M.C have stocks in ADEL, Inc.

\section{References}

1. Braak, H.; Braak, E. Neuropathological stageing of Alzheimer-related changes. Acta Neuropathol. 1991;82(4):239-259.

2. de Calignon, A.; Polydoro, M.; Suarez-Calvet, M.; William, C.; Adamowicz, D.H.; Kopeikina, K.J.; Pitstick, R.; Sahara, N.; Ashe, K.H.; Carlson, G.A.; Spires-Jones, T.L.; Hyman, B.T. Propagation of tau pathology in a model of early Alzheimer's disease. Neuron. 2012 Feb 23;73(4):685-697.

3. Michel, C.H.; Kumar, S.; Pinotsi, D.; Tunnacliffe, A.; St George-Hyslop, P.; Mandelkow, E.; Mandelkow, E.M.; Kaminski, C.F.; Kaminski Schierle, G.S. Extracellular monomeric tau protein is sufficient to initiate the spread of tau protein pathology. J Biol Chem. 2014 Jan 10;289(2):956-967.

4. Wu, J.W.; Hussaini, S.A.; Bastille, I.M.; Rodriguez, G.A.; Mrejeru, A.; Rilett, K.; Sanders, D.W.; Cook, C.; Fu, H.; Boonen, R.A.; Herman, M.; Nahmani, E.; Emrani, S.; Figueroa, Y.H.; Diamond, M.I.; Clelland, C.L.; Wray, S.; Duff, K.E. Neuronal activity enhances tau propagation and tau pathology in vivo. Nat Neurosci. 2016 Aug;19(8):1085-1092.

5. Clavaguera, F.; Bolmont, T.; Crowther, R.A.; Abramowski, D.; Frank, S.; Probst, A.; Fraser, G.; Stalder, A.K.; Beibel, M.; Staufenbiel, M.; Jucker, M.; Goedert, M.; Tolnay, M. Transmission and spreading of tauopathy in transgenic mouse brain. Nat Cell Biol. 2009 Jul;11(7):909-913.

6. Goedert, M.; Spillantini, M.G.; Jakes, R.; Rutherford, D.; Crowther, R.A. Multiple isoforms of human microtubule-associated protein tau: sequences and localization in neurofibrillary tangles of Alzheimer's disease. Neuron. 1989 Oct;3(4):519-526.

7. Irwin, D.J.; Cohen, T.J.; Grossman, M.; Arnold, S.E.; Xie, S.X.; Lee, V.M.; Trojanowski, J.Q. Acetylated tau, a novel pathological signature in Alzheimer's disease and other tauopathies. Brain. 2012 Mar;135(Pt 3):807-818.

8. Min, S.W.; Cho, S.H.; Zhou, Y.; Schroeder, S.; Haroutunian, V.; Seeley, W.W.; Huang, E.J.; Shen, Y.; Masliah, E.; Mukherjee, C.; Meyers, D.; Cole, P.A.; Ott, M.; Gan, L. Acetylation of tau inhibits its degradation and contributes to tauopathy. Neuron. 2010 Sep 23;67(6):953-966.

9. Cohen, T.J.; Guo, J.L.; Hurtado, D.E.; Kwong, L.K.; Mills, I.P.; Trojanowski, J.Q.; Lee, V.M. The acetylation of tau inhibits its function and promotes pathological tau aggregation. Nat Commun. 
2011;2:252.

10. Momeni, P.; Pittman, A.; Lashley, T.; Vandrovcova, J.; Malzer, E.; Luk, C.; Hulette, C.; Lees, A.; Revesz, T.; Hardy, J.; de Silva, R. Clinical and pathological features of an Alzheimer's disease patient with the MAPT Delta K280 mutation. Neurobiol Aging. 2009 Mar;30(3):388-393.

11. Rizzu, P.; Van Swieten, J.C.; Joosse, M.; Hasegawa, M.; Stevens, M.; Tibben, A.; Niermeijer, M.F.; Hillebrand, M.; Ravid, R.; Oostra, B.A.; Goedert, M.; van Duijn, C.M.; Heutink, P. High prevalence of mutations in the microtubule-associated protein tau in a population study of frontotemporal dementia in the Netherlands. Am J Hum Genet. 1999 Feb;64(2):414-421.

12. Mocanu, M.M.; Nissen, A.; Eckermann, K.; Khlistunova, I.; Biernat, J.; Drexler, D.; Petrova, O.; Schonig, K.; Bujard, H.; Mandelkow, E.; Zhou, L.; Rune, G.; Mandelkow, E.M. The potential for beta-structure in the repeat domain of tau protein determines aggregation, synaptic decay, neuronal loss, and coassembly with endogenous Tau in inducible mouse models of tauopathy. J Neurosci. $2008 \mathrm{Jan}$ 16;28(3):737-748.

13. Mukrasch, M.D.; Biernat, J.; von Bergen, M.; Griesinger, C.; Mandelkow, E.; Zweckstetter, M. Sites of tau important for aggregation populate \{beta\}-structure and bind to microtubules and polyanions. $\mathrm{J}$ Biol Chem. 2005 Jul 1;280(26):24978-24986.

14. Vorhees, C.V.W., M.T. Morris water maze: procedures for assessing spatial and related forms of learning and memory. . Nat Protoc. 2006;1, 848-858.

15. Hirotaka Shoji1, Keizo Takao2,3, Satoko Hattori1,2, Tsuyoshi Miyakawa1,2,3. Contextual and Cued Fear Conditioning Test Using a Video Analyzing System in Mice. Journal of Visualized Experiments. 2014.

16. H-J Kim, M.-H.C., WH Shim, JK Kim, E-Y Jeon, D-H Kim and S-Y Yoon. Deficient autophagy in microglia impairs synaptic pruning and causes social behavioral defects. Molecular Psychiatry. 2017 (22):1576-1584.

17. Kaoru Yamada, T.K.P., Katja Hochgräfe, Thomas E. Mahan, Hong Jiang, Floy R. Stewart,; Holtzman, E.-M.M.a.D.M. Analysis of in vivo turnover of tau in a mouse model of tauopathy. Molecular Neurodegeneration. 2015;10:55.

18. Sigurdsson, E.E.C.a.E.M. Tau-targeting therapies for Alzheimer disease. Nat Rev Neurol. 2018;14(7): 399-415.

19. Kontaxi, C.; Piccardo, P.; Gill, A.C. Lysine-Directed Post-translational Modifications of Tau Protein in Alzheimer's Disease and Related Tauopathies. Front Mol Biosci. 2017;4:56.

20. Wong, H.K.; Veremeyko, T.; Patel, N.; Lemere, C.A.; Walsh, D.M.; Esau, C.; Vanderburg, C.; Krichevsky, A.M. De-repression of FOXO3a death axis by microRNA-132 and -212 causes neuronal apoptosis in Alzheimer's disease. Hum Mol Genet. 2013 Aug 1;22(15):3077-3092.

21. Aubry, S.; Shin, W.; Crary, J.F.; Lefort, R.; Qureshi, Y.H.; Lefebvre, C.; Califano, A.; Shelanski, M.L. Assembly and interrogation of Alzheimer's disease genetic networks reveal novel regulators of progression. PLoS One. 2015;10(3):e0120352. 
22. Min, S.W.; Chen, X.; Tracy, T.E.; Li, Y.; Zhou, Y.; Wang, C.; Shirakawa, K.; Minami, S.S.; Defensor, E.; Mok, S.A.; Sohn, P.D.; Schilling, B.; Cong, X.; Ellerby, L.; Gibson, B.W.; Johnson, J.; Krogan, N.; Shamloo, M.; Gestwicki, J.; Masliah, E.; Verdin, E.; Gan, L. Critical role of acetylation in tau-mediated neurodegeneration and cognitive deficits. Nat Med. 2015 Oct;21(10):1154-1162.

23. Chen, X.; Li, Y.; Wang, C.; Tang, Y.; Mok, S.-A.; Tsai, R.M.; Rojas, J.C.; Karydas, A.; Miller, B.L.; Boxer, A.L.; Gestwicki, J.E.; Cuervo, A.M.; Arkin, M.; Gan, L. Tau Secretion and Propagation Is Regulated by p300/CBP via Autophagy-Lysosomal Pathway in Tauopathy. bioRxiv. 2018:418640.

24. Fitzpatrick, A.W.P.; Falcon, B.; He, S.; Murzin, A.G.; Murshudov, G.; Garringer, H.J.; Crowther, R.A.; Ghetti, B.; Goedert, M.; Scheres, S.H.W. Cryo-EM structures of tau filaments from Alzheimer's disease. Nature. 2017 Jul 13;547(7662):185-190.

25. Seidler, P.M.; Boyer, D.R.; Rodriguez, J.A.; Sawaya, M.R.; Cascio, D.; Murray, K.; Gonen, T.; Eisenberg, D.S. Structure-based inhibitors of tau aggregation. Nat Chem. 2018 Feb;10(2):170-176.

26. Courade, J.P.; Angers, R.; Mairet-Coello, G.; Pacico, N.; Tyson, K.; Lightwood, D.; Munro, R.; McMillan, D.; Griffin, R.; Baker, T.; Starkie, D.; Nan, R.; Westwood, M.; Mushikiwabo, M.L.; Jung, S.; Odede, G.; Sweeney, B.; Popplewell, A.; Burgess, G.; Downey, P.; Citron, M. Epitope determines efficacy of therapeutic anti-Tau antibodies in a functional assay with human Alzheimer Tau. Acta Neuropathol. 2018 Nov;136(5):729-745.

\section{Figures}


A

A

Wild type

: Adjuvant
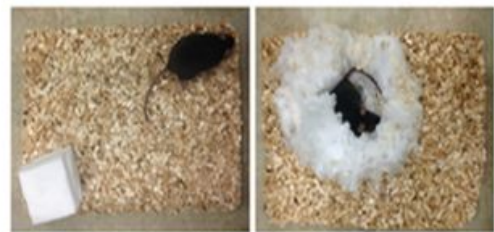

Tau P301L : Adjuvant
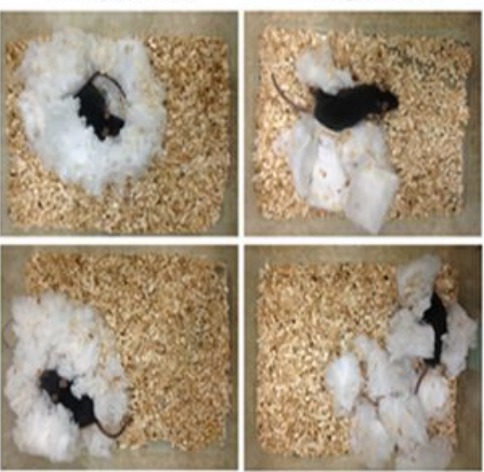

Tau P301L : acK280

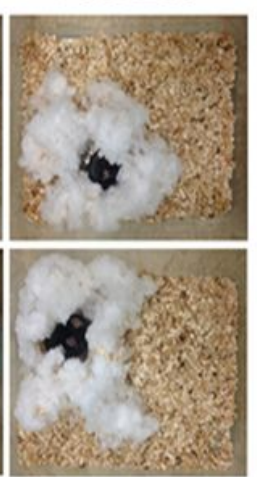

C

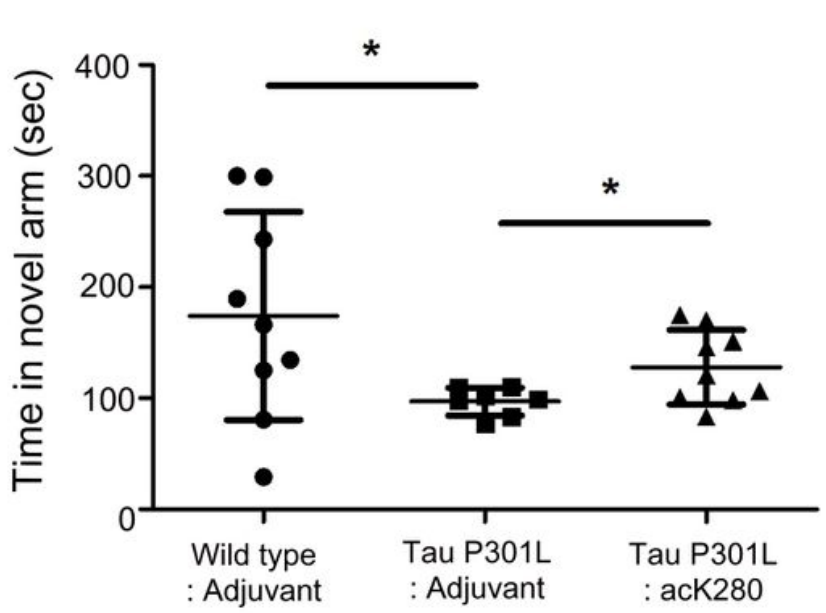

B

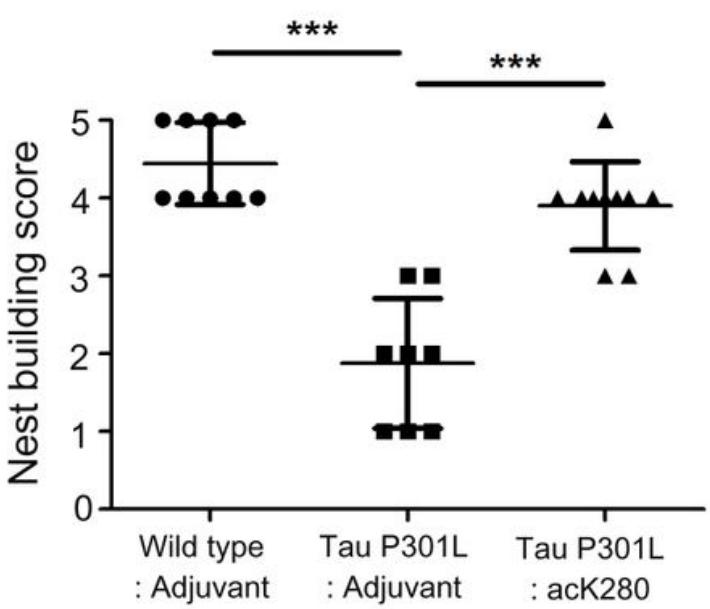

D

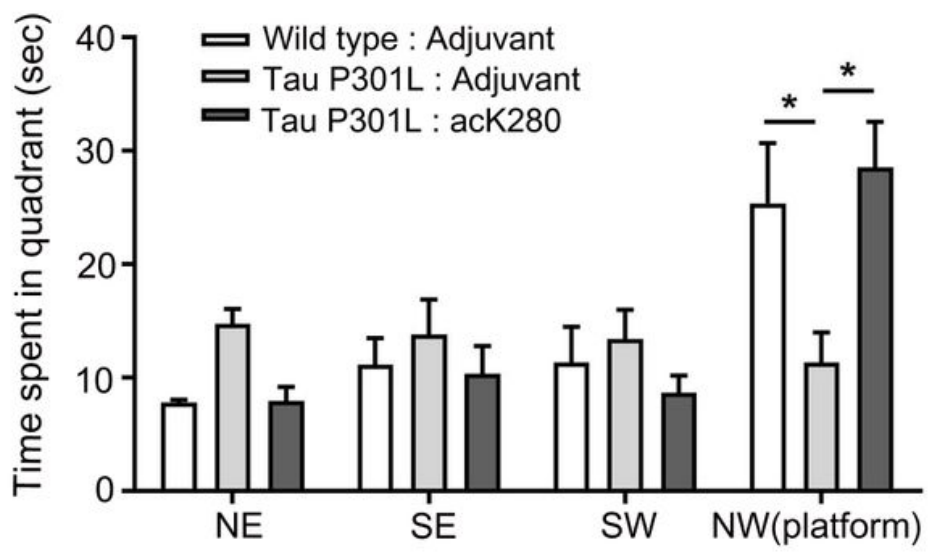

Figure 1

Vaccination with acetylated tau at K280 ameliorates cognition in tau-Tg mice. Representative analyzed data: acK280 peptide results in A-B nest building test C Y-maze and D Morris water-maze. Wild typeadjuvant n=9, Tau P301L-adjuvant n=8, Tau P301L-acK280 n=9, all mice are male. NE: North East, SE: South East, SW: South West, NW: North West quadrants. ${ }^{*} \mathrm{P}<0.05,{ }^{\star \star \star} \mathrm{P}<0.001$ compared to Tau P301Ladjuvant group. 
A

C

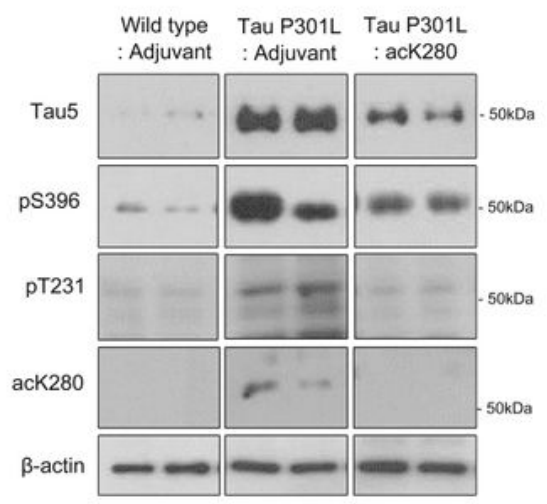

D
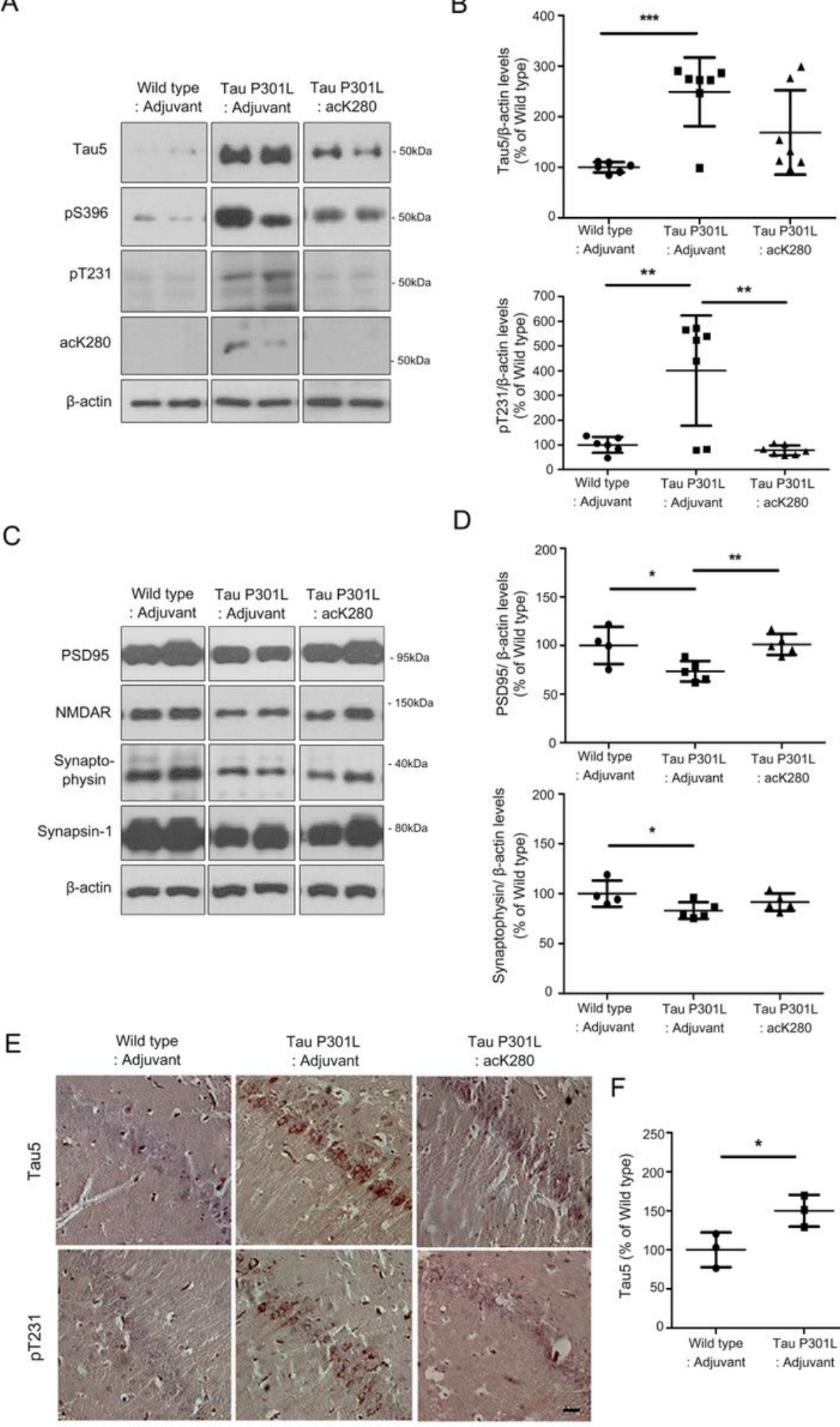
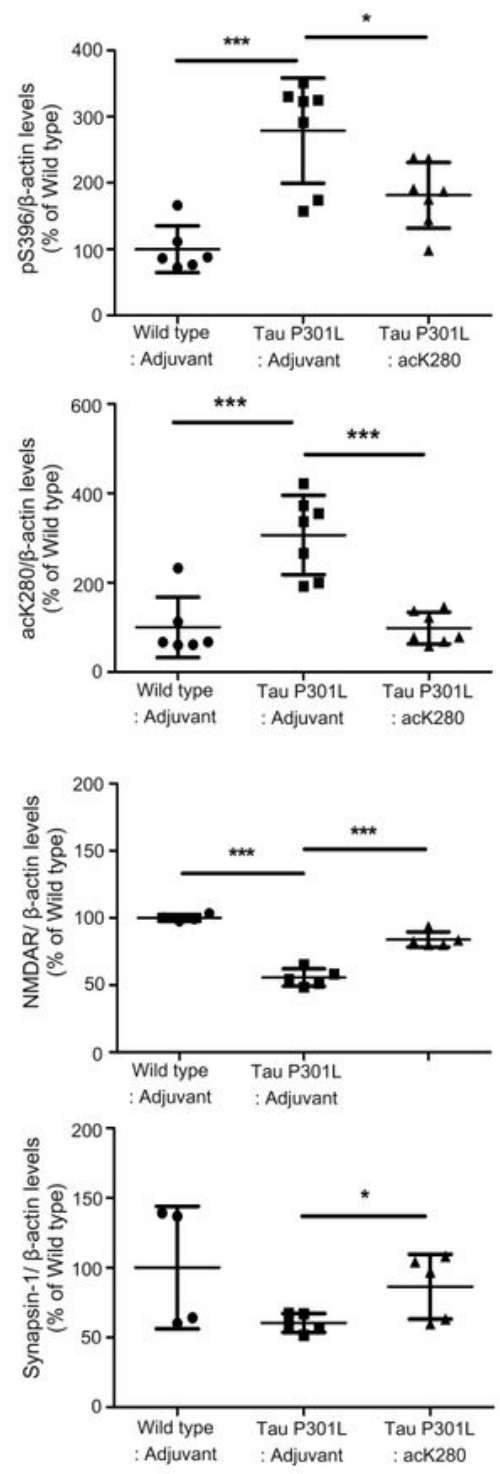

$F$
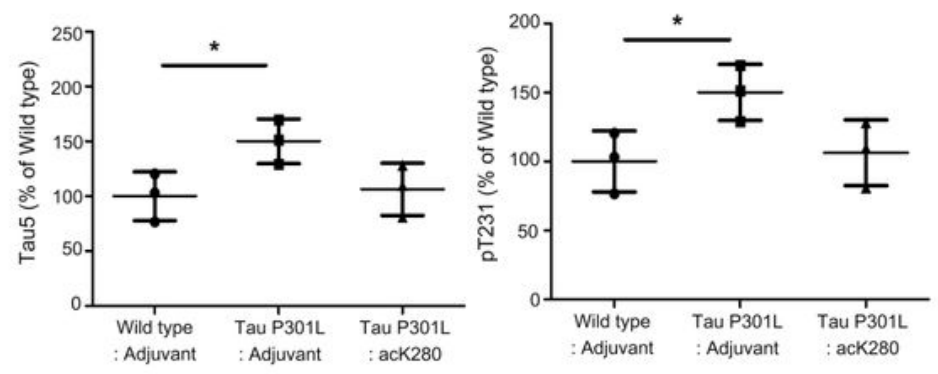

\section{Figure 2}

Ameliorated pathology by Tau-acK280 active immunization. A Representative immunoblots of Tau5, pS396, pT231, acK280 (Anaspec), and $\beta$-actin in the cortex from 8-month-old immunized mice (cropped blot images are shown, see Additional file 7: Figure S7 for full immunoblots). B Quantification of Tau5, pSer396, pT231, and acK280 (Anaspec) protein levels normalized to $\beta$-actin levels. Wild type-adjuvant $n=6$, Tau P301L-adjuvant n=7, Tau P301L-acK280 n=7, all mice are male. *P $<0.05$, **P $<0.01$, ***P $<$ 
0.001 compared to Tau P301L-adjuvant group. C Representative immunoblots of PSD95, NMDAR, synaptophysin, synapsin-1, and $\beta$-actin in the cortex from 8-month-old immunized mice (cropped blot images are shown, see Additional file 7: Figure S7 for full immunoblots). D Quantification of PSD95, NMDAR, synaptophysin, and synapsin-1 protein levels normalized to $\beta$-actin levels. Wild type-adjuvant $n=4$, Tau P301L-adjuvant $n=5$, Tau P301L-acK280 n=5, all mice are male. ${ }^{*} P<0.05, * * P<0.01, * * * P<$ 0.001 compared to Tau P301L-adjuvant group. E Representative immunostaining of Tau5 and pT231 in brains of 8-month-old immunized mice. F Quantification of Tau5 and pT231 levels. Wild type-adjuvant $\mathrm{n}=3$, Tau P301L-adjuvant $\mathrm{n}=3$, Tau P301L-ack280 n=3, all mice are male. ${ }^{*} \mathrm{P}<0.05$ compared to Tau P301L-adjuvant group.

A

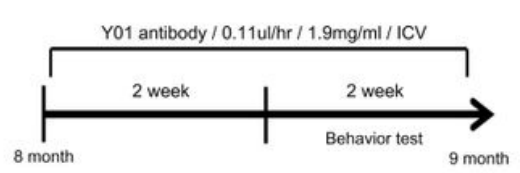

C

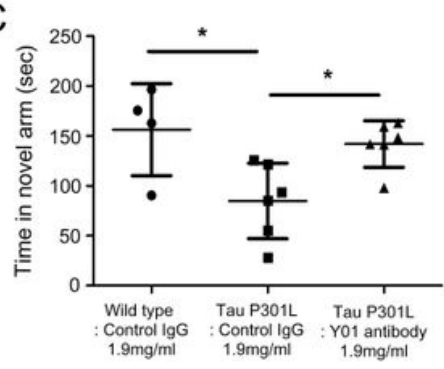

F

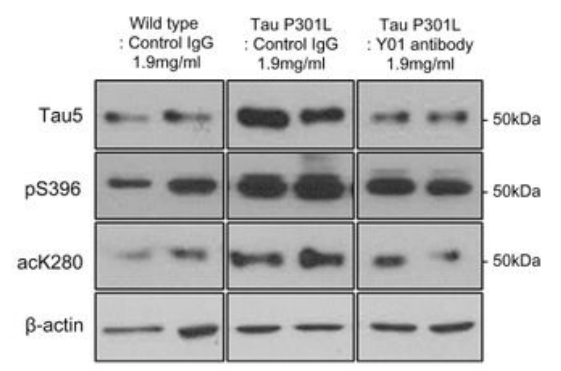

H

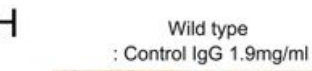
Control lgG $1.9 \mathrm{mg} / \mathrm{ml}$
B

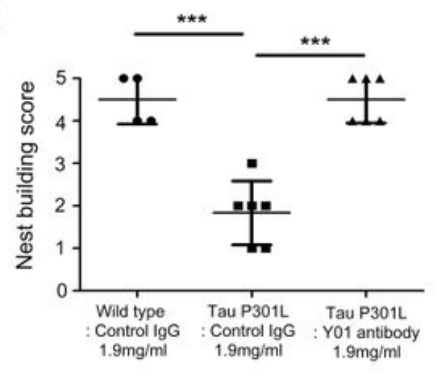

D

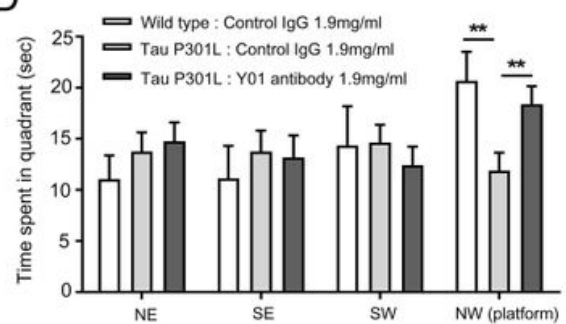

G

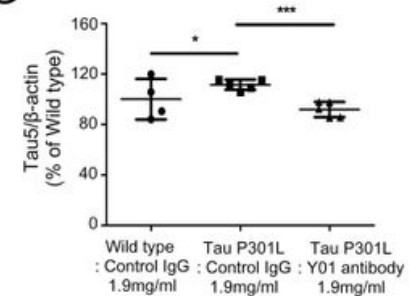

E
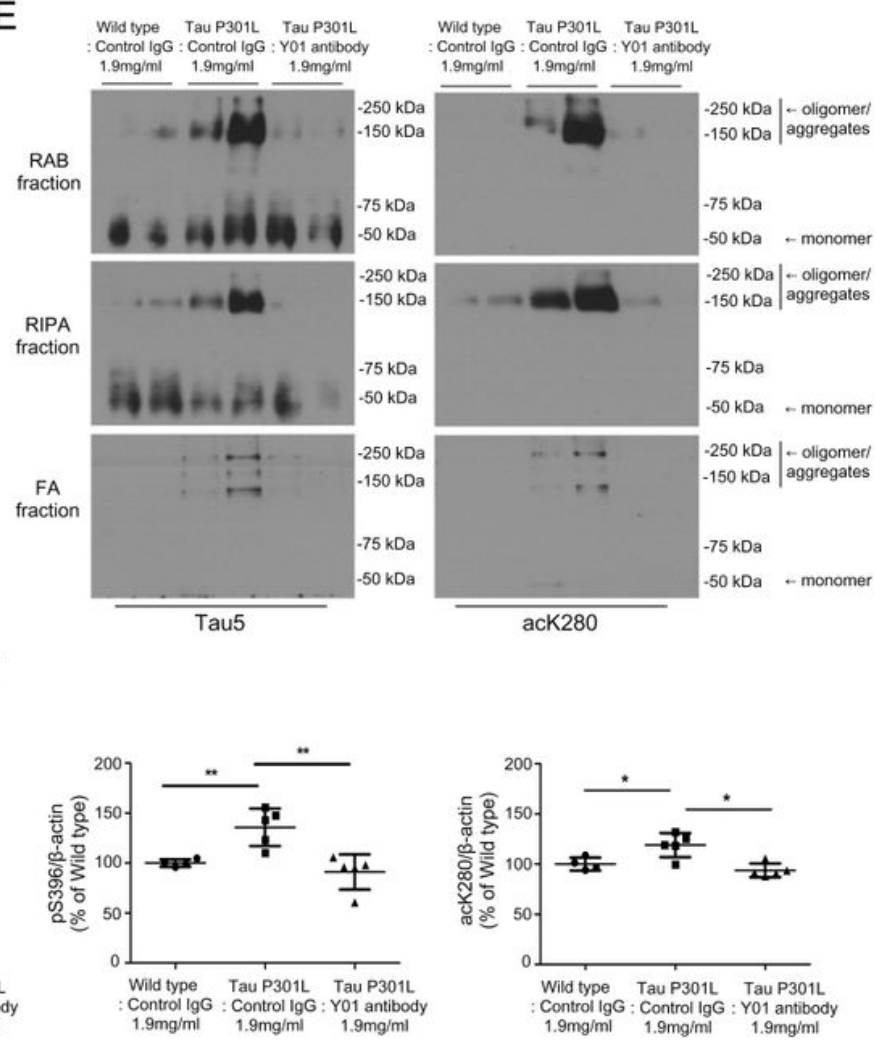

Tau P301L : Y01 antibody $1.9 \mathrm{mg} / \mathrm{ml}$

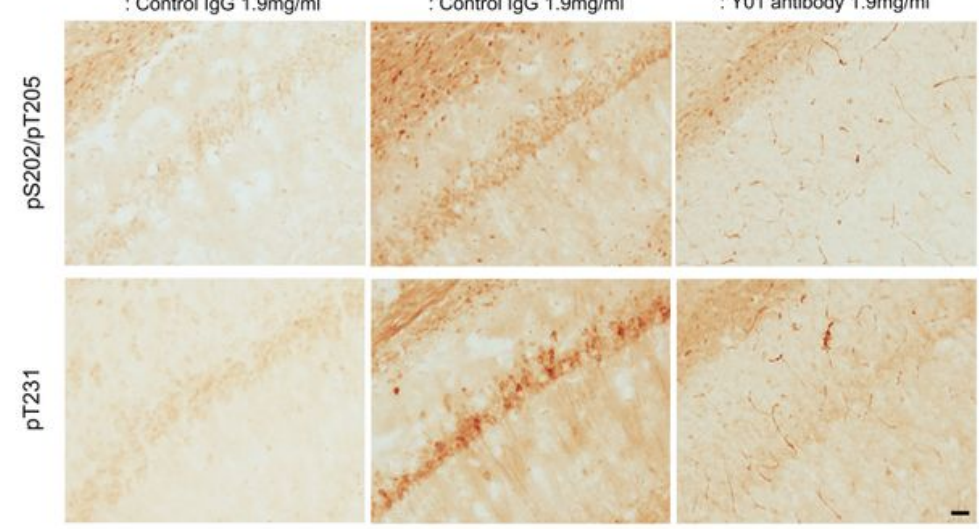

I
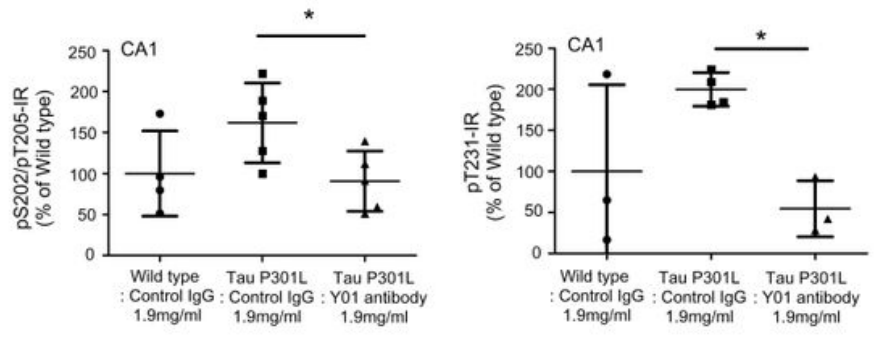

Figure 3

Ameliorated cognition and pathology by intracerebroventricular infusion of Y01 antibody. A Schematic diagram of the passive immunization experimental design. ICV: intracerebroventricular infusion. B-D After 
two weeks of starting infusions, behavioral tests were performed during the next 2 weeks of infusion. B nest building test C Y-maze D Morris water maze. Wild type-Control IgG $n=4$, Tau P301L-Control IgG $n=6$, Tau P301L-Y01 antibody n=6, all mice are male. NE: North East, SE: South East, SW: South West, NW: North West quadrants. E Representative immunoblots of Tau5 and Tau-acK280 in RAB fractions, RIPA fractions, and FA fractions of the cortex from immunized mice. Wild type-Control IgG n=2, Tau P301LControl IgG n=2, Tau P301L-Y01 antibody $n=2$, all mice are male. F Representative immunoblots of Tau5, Tau-pS396, and Tau-acK280 in the cortex from immunized mice (cropped blot images are shown, see Additional file 7: Figure S7 for full immunoblots). G Quantification of Tau5, Tau-pS396, and Tau-ack280 protein levels normalized to $\beta$-actin. Wild type-Control IgG $n=4$, Tau P301 L-Control IgG $n=5$, Tau P301LY01 antibody $n=5$, all mice are male. ${ }^{\star} \mathrm{P}<0.05,{ }^{* *} \mathrm{P}<0.01$, ${ }^{\star \star *} \mathrm{P}<0.001$ compared to Tau P301L-Control IgG group. H Representative immunohistochemistry of Tau-pS202/pT205 and pT231 in CA1 of mouse hippocampus. I Quantification of Tau-pS202/pT205 levels in CA1 region. Wild type-Control IgG $n=4$, Tau P301L-Control IgG n=5, Tau P301L-Y01 antibody n=5. Quantification of pT231 levels in CA1 region. Wild type-Control IgG $n=3$, Tau P301L-Control IgG $n=4$, Tau P301L-Y01 antibody $n=3$, all mice are male. * $P<$ 0.05 compared to Tau P301L-Control IgG group. 
A

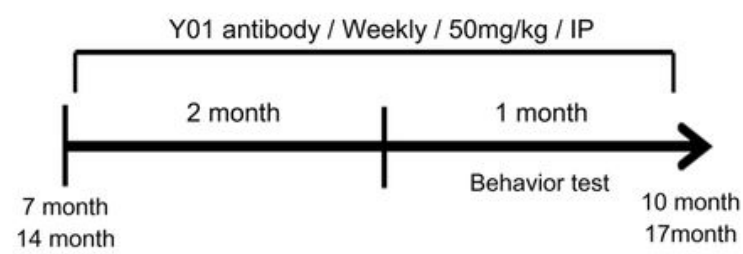

C

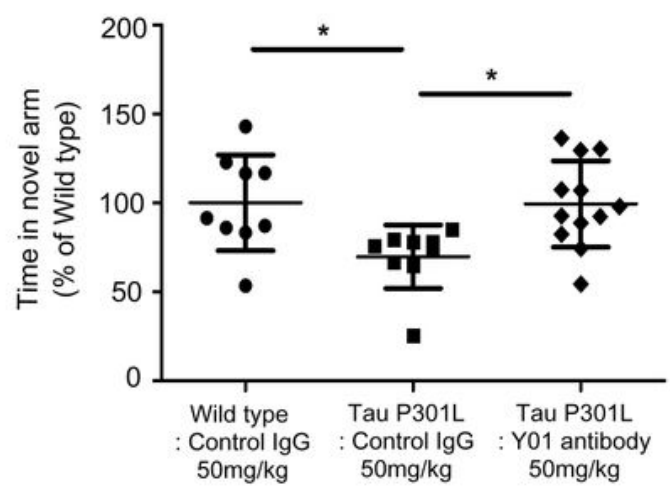

D

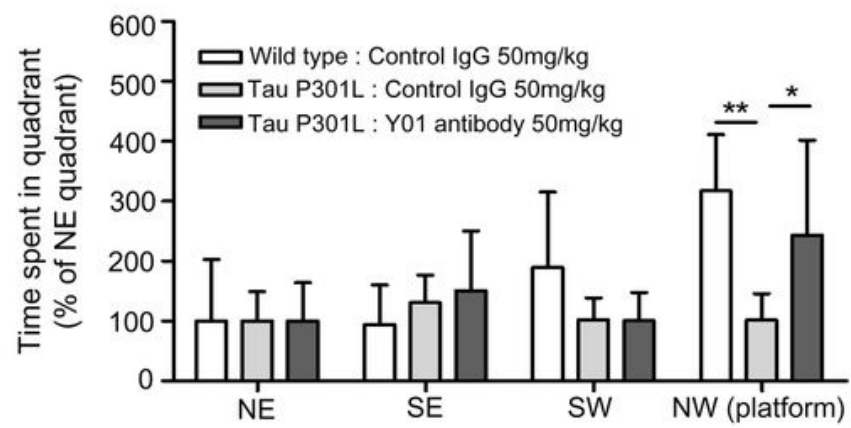

$E$

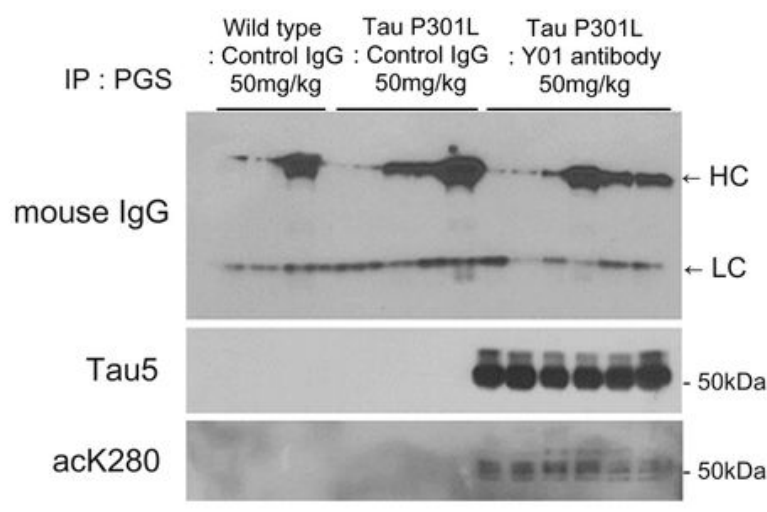

B
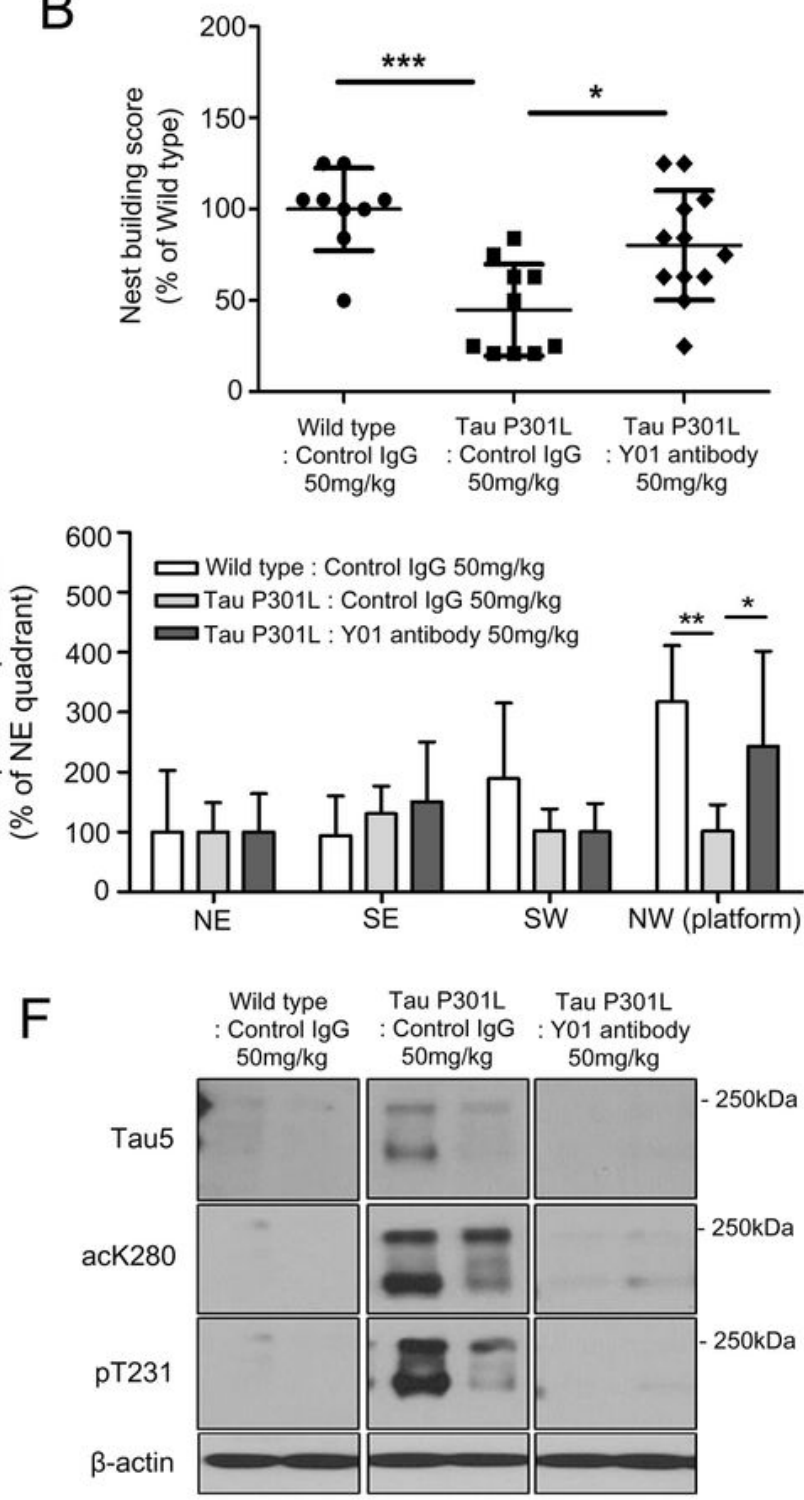

G
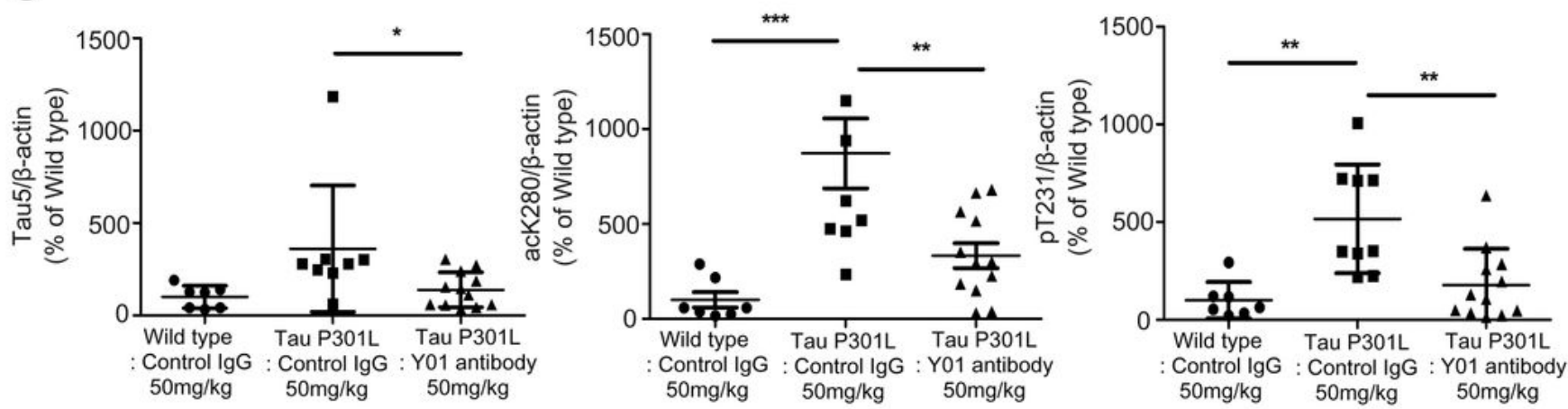

\section{Figure 4}

Ameliorated cognition and pathology by intraperitoneal injection of Y01 antibody. A Schematic diagram of the passive immunization experimental design (7 and 14 months old). IP: intraperitoneal injection. B-D After Control IgG or Y01 antibody weekly injections during two months, behavioral tests were performed during the next 1 month of weekly injections. B nest building test C Y-maze D Morris water maze. Wild type-Control IgG n=9, Tau P301L-Control IgG n=10, Tau P301L-Tau-Y01 antibody n=12. NE: North East, 
SE: South East, SW: South West, NW: North West quadrants. ${ }^{\star} P<0.05,{ }^{\star} * \mathrm{P}<0.01, \star \star \star P<0.001$ compared to Tau P301L-Control IgG group. E Representative immunoblots of mouse lgG (mlgG), Tau5, and TauacK280 proteins co-immunoprecipitated with the protein-G sepharose (PGS) from the Control IgG or Y01 antibody-injected mouse cortex (cropped blot images are shown, see Additional file 8: Figure S8 for full immunoblots). HC arrow: heavy chain, LC arrow: light chain. F Representative semi-denatured immunoblots of Tau5, Tau-acK280, and pT231 protein levels (cropped blot images are shown, see Additional file 8: Figure S8 for full immunoblots). G Quantification of Tau5, Tau-acK280, and pT231 protein levels normalized to $\beta$-actin. Wild type-Control IgG n=7, Tau P301L-Control IgG n=9, Tau P301LY01 antibody $n=12$. ${ }^{*} P<0.05, * * P<0.01$, ***P $<0.001$ compared to Tau P301L-Control IgG group.

A

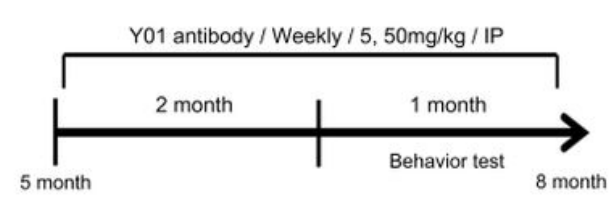

B

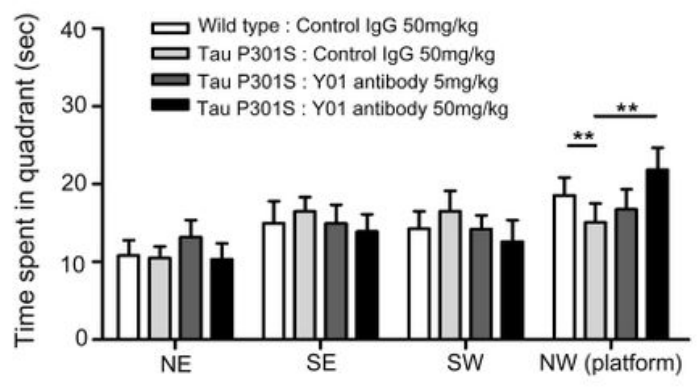

C

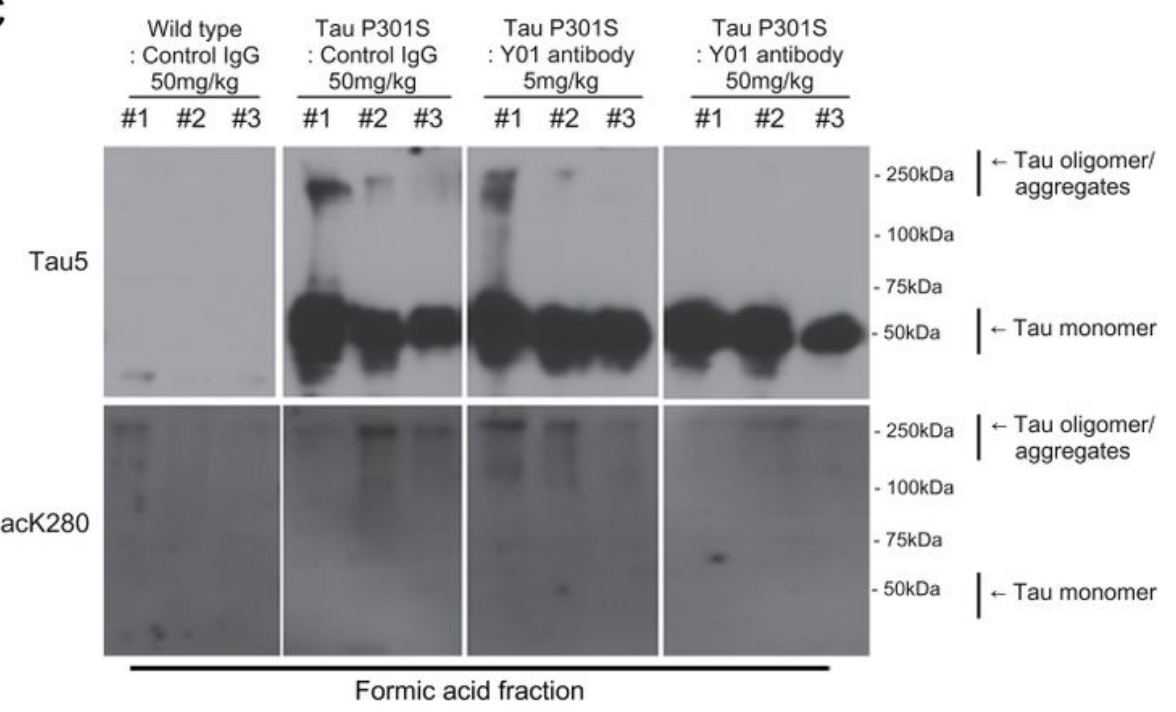

D
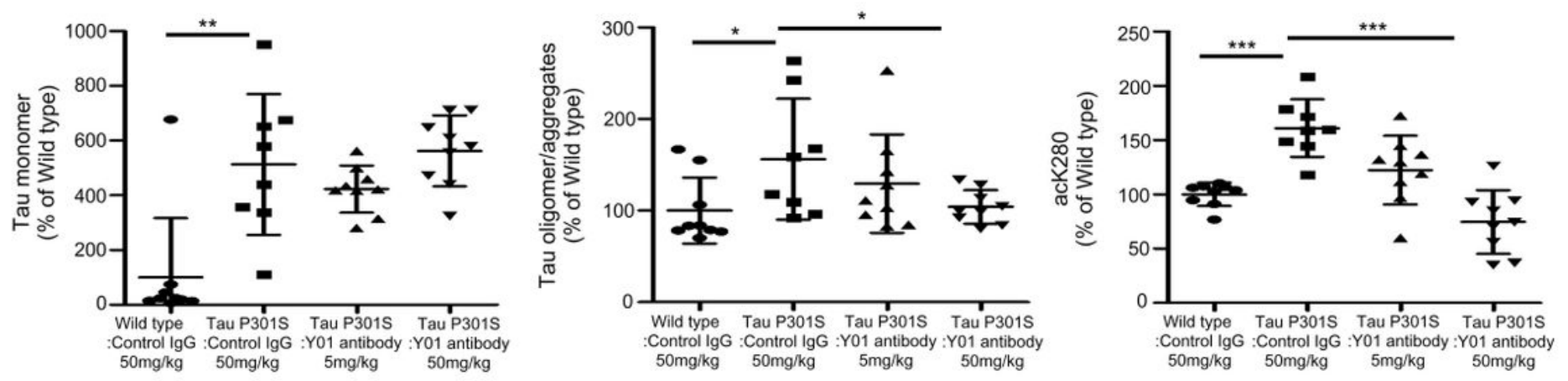

\section{Figure 5}

Ameliorated cognition and pathology by intraperitoneal injection of Y01 antibody in Tau P301S mice. A Schematic diagram of the passive immunization experimental design. IP: intraperitoneal injection. After Control IgG or Y01 antibody weekly injections during two months, behavioral test was performed during the next one month of weekly injection. B Morris water maze. Wild type-Control $\lg G n=11$ (female $n=4$, male $n=7$ ), Tau P301S-Control IgG $n=15$ (female $n=6$, male $n=9$ ), Tau P301S-Y01 antibody $5 \mathrm{mg} / \mathrm{kg} \mathrm{n=13}$ (female $\mathrm{n}=5$, male $\mathrm{n}=8$ ), Tau P301S-Y01 antibody 50mg/kg n=13 (female $\mathrm{n}=4$, male $\mathrm{n}=9$ ). Unpaired twotailed Student's t-tests. ${ }^{*} \mathrm{P}<0.01$ compared to Tau P301S-Control IgG group C Representative semidenatured immunoblots of Tau 5 and acK280 protein in the hippocampus from 8-month passive immunized mice (cropped blot images are shown, see Additional file 8: Figure S8 for full immunoblots). D 
Quantification of Tau monomer, Tau oligomer/aggregates and acK280 protein levels normalized to GAPDH. Wild type-Control IgG $n=9$ (female $n=4$, male $n=5$ ), Tau P301S-Control lgG $n=8$ (female $n=3$, male $\mathrm{n}=5$ ), Tau P301S-Y01 antibody $5 \mathrm{mg} / \mathrm{kg} \mathrm{n}=9$ (female $\mathrm{n}=4$, male $\mathrm{n}=5$ ), Tau P301S-Y01 antibody 50mg/kg $n=9$ (female $n=2$, male $n=7$ ). ${ }^{*}<<0.05, * * P<0.01, * \star * P<0.001$ compared to Tau P301S-Control IgG group.

\section{Supplementary Files}

This is a list of supplementary files associated with this preprint. Click to download.

- SupplementaryMaterial.docx 